\title{
Prevalence of HPV among HIV-negative women of child-bearing age in Lomé, Togo
}

\author{
Yawo T Nyasenu*,1 (D), Fifonsi A Gbeasor-Komlanvi2,3 (D), Sabi Abdul-Raouf Issa1, Amivi \\ Ehlan ${ }^{1}$, Martin K Tchankoni' ${ }^{3}$, Brice M Yambiyo ${ }^{4}$, Mireille Prince-David', Mounerou Salou', \\ Didier K Ekouevi ${ }^{2,3,5,6}$ \& Anoumou Dagnra ${ }^{1}$ \\ ${ }^{1}$ Département des Sciences Fondamentales, Laboratoire de Biologie Moléculaire et d'Immunologie (BIOLIM), Université de Lomé, \\ Lomé, Togo \\ ${ }^{2}$ Département de Santé Publique, Université de Lomé, Lomé, Togo \\ ${ }^{3}$ Centre Africain de Recherche en Epidémiologie et en Santé Publique, Lomé, Togo \\ ${ }^{4}$ Service d'Epidémiologie, Institut Pasteur de Bangui, Bangui, République Centrafricaine \\ ${ }^{5}$ Institut de Santé Publique Epidémiologie Développement (ISPED), Université de Bordeaux, Bordeaux, France \\ ${ }^{6}$ Centre Inserm, 1219, Université de Bordeaux, Bordeaux, France \\ *Author for correspondence: nyasenu@yahoo.fr
}

\begin{abstract}
Aim: This study aimed to assess the prevalence and the distribution of HPV genotypes among HIV-negative women of child-bearing age in Lomé, Togo. Materials \& methods: From April 2014 to September 2015, a cross-sectional study was conducted among HIV-negative women attending gynecological consultation in six health centers in Lomé. Cervical swabs were obtained from 324 women. HPV test was performed using HPV Direct Flow Chip. Results: The prevalence of any type and oncogenic HPV was 9.3 and $8.3 \%$, respectively. A total of 13 different genotypes HPV, high risk (16, 18, 35, 45, 52, 53, 68, 82) and low risk $(6,40,43,44 / 65,62 / 81)$, were found. Conclusion: Findings from this study provide essential insights for planning future public health strategies, including HPV vaccination programs.
\end{abstract}

First draft submitted: 31 October 2019; Accepted for publication: 9 December 2019; Published online: 20 December 2019

Keywords: HIV-negative women • HPV genotyping • human papillomavirus • Lomé, Togo • oncogenic HPV • prevalence

The association between cervical cancer and HPV is well known. HPV is the most common cause of cervical cancer, which is the second most frequent female cancer worldwide [1]. HPV is mainly transmitted through sexual contact and most people are infected with HPV shortly after the onset of sexual activity [2]. Cervical cancer is caused by sexually acquired infection with certain types of HPV [2,3]. In 2018, cervical cancer was the fourth most frequent cancer in women with an estimated 570,000 new cases worldwide and represented $7.5 \%$ of all female cancer deaths. Every year, more than $85 \%$ of cervical cancer-related deaths occur in less developed regions [2].

More than 200 genotypes of HPV have been described. Some HPV are classified as low-risk oncogenic HPV (LR-HPV), which cause papilloma and condyloma acuminata. High-risk oncogenic HPV (HR-HPV) are cancer causing [2]. HPV types 16 and 18 account for nearly $70 \%$ of all cases of invasive cervical cancer worldwide. This percentage rises to $90 \%$ for HPV6/11/16/18/31/33/45/52/58 [3]. Main factors associated with HPV are: multiple sexual partners, early sexual activity, prolonged oral contraception use, smoking, poor socio-economic conditions, associated sexually transmitted infections and HIV infection [3-5].

The burden of cervical cancer and HPV infection can be reduced and control strategies rely on HPV vaccination and early detection of benign or precancerous cervical lesions [5]. To date, three HPV vaccines are widely marketed internationally: Cervarix ${ }^{\circledR}$ (GlaxoSmithKline), Gardasil ${ }^{\circledR}$ (Merck), and Gardasil- ${ }^{\circledR}{ }^{\circledR}$ (Merck), which protect against HPV 16/18, HPV 6/11/16/18 and HPV-6/11/16/18/31/33/45/52/58, respectively [6,7]. These genotypes, generally identified in more than $70 \%$ of cervical cancer cases worldwide, are still relevant in most African countries, while others not included in the vaccines have been found to be circulating and might be contributing to clinical conditions $[8,9]$. Thus, describing HPV type circulation in Togo is needed to inform vaccination program.

Future Medicine 
In Togo, cervical cancer is a public health problem and it is the second most common cancer in women [10], with an estimated mortality rate of $12.5 \%$ in 2018 in Togo [2]. Few data are available on circulating genotypes of HPV among key populations and women infected with HIV in the country [8,11,12]. However, no data are available on HPV prevalence in the general population, while Togo had received funding from the Global Alliance for Vaccines and Immunization to carry out a demonstration project for the administration of HPV vaccine, in two districts (Tchamba and Golfe) for 2 school years (2015-2016, 2017-2018). It is important to have essential data for planning future public health strategies, including HPV vaccination programs. The present study aimed to estimate the prevalence of HPV and to describe the distribution of HPV genotypes among HIV-negative women of child-bearing age in Lomé, Togo.

\section{Materials \& methods}

\section{Study design \& setting}

A cross-sectional study was conducted over a period of 18 months (from April 2014 to September 2015) among women attending gynecological consultation in six health centers in Lomé: three public health clinics (the department of Histology-Embryology of the teaching hospital 'Centre Hospitalier Universitaire Sylvanus Olympio', the 'Centre Médical Social Agoe' and the 'Centre Médical Social Adidogomé’) and three private clinics (Alpia, Biasa and Wossinu-Gbogbo).

\section{Sample size \& participants}

We included all HIV-negative women, aged 18 years and older, who came for a gynecological visit during the study period. Women who gave their approval for the HPV cervical sampling and gave their informed written consent were recruited.

Since no data on HPV infection were available in the general population in Togo, the sample size calculation was based on the following assumptions: an expected prevalence of HPV infection of $30 \%$ based on estimates in a population of HIV-negative women of child-bearing age of 33\% in Côte d'Ivoire [5], country of West Africa, with a precision of $6 \%$, a significance level set at $5 \%$ and a non-response rate of $10 \%$; the minimum sample size was estimated at 246 participants.

\section{Data collection}

A standardized questionnaire was used to collect relevant sociodemographic, clinical and biological information during a face-to-face interview.

A cytobrush (Hospitex Diagnostics Srl) was used to collect the cells at the junction between the endocervix and the ectocervix and these cells were stored in a preservative solution provided by the manufacturer (Cytofast solution 42010600, Hospitex Diagnostics Srl, Sesto Fiorentino, Firenze, Italy). Cervical cells were transported and stored at ambient temperature $\left(10-30^{\circ} \mathrm{C}\right)$ for a maximum time period of 5 days until manipulation at the 'Laboratoire de Biologie Moléculaire et Immunologie' of the 'Faculté des Sciences de la Santé of the Université de Lomé'.

For the HPV testing, specimens were analyzed by the HPV Direct Flow Chip system, which is intended for simultaneous detection and genotyping of $36 \mathrm{HPV}$ types (high-risk HPV [16, 18, 26, 31, 33, 35, 39, 45, 51, 52, 53, 56, 58, 59, 66, 68, 73 and 82] and low-risk HPV [6, 11, 40, 42, 43, 44, 54, 55, 61, 62, 67, 69, 70, 71, 72, 81, 84 and 89] [=CP6108]), by direct PCR, followed by reverse dot blot automatic hybridization, based on DNA-flow technology and colorimetric detection with the automatic e-BRID ${ }^{\circledR}$ System [13]. The amplification was carried out by PCR from crude-cell extracts, by using the PCR Mix and Phire Hot Start II DNA Polymerase (MAD003930MU-P-E-30, Master Diagnostica, Granada, Spain). Amplicons were hybridized, using the optimized HPV Direct Flow Chip kit (MAD-003930M-H, Master Diagnostica) [14]. HPV chip membranes contained immobilized probes for hybridization control, beta-globin gene (for quality control of amplification), HPV-consensus sequences and genotype-specific HPV detection. The hybridization was performed automatically in sets of 15 samples using the e-BRID System (Master Diagnostica) [13,14]. HPV Direct Flow Chip had a sensibility of 100\% and a specificity of 100\% [15]. HPV Direct Flow Chip results were comparable with those of the other HPV tests like Linear Array HPV Genotyping Test, CLART ${ }^{\circledR}$ HPV2 and Digene ${ }^{\circledR}$ Hybrid Capture 2 HPV DNA Test [14].

\section{Statistical analyses}

Data were entered into a Microsoft Excel database. The Chi-squared test or the exact Fisher test and the MannWhitney U test or the Wilcoxon test, were used for comparison of categorical and quantitative variables, respectively. 
Table 1. Socio-demographic, clinical and biological characteristics.

\begin{tabular}{|lll|}
\hline Characteristics & $\mathbf{N}(\mathbf{n}=324)$ & $\%$ \\
\hline Age (years) & 89 & 27.4 \\
\hline $20-29$ & 80 & 24.6 \\
\hline $30-39$ & 102 & 31.4 \\
\hline $40-49$ & 53 & 16.3 \\
\hline$\geq 50$ & 164 & 50.6 \\
\hline Marital status & 160 & 49.4 \\
\hline Lives alone & & 25.0 \\
\hline Married/cohabiting & 81 & 47.5 \\
\hline Education level & 154 & 27.5 \\
\hline None or primary school & 89 & \\
\hline Secondary school & & 63.5 \\
\hline University & 206 & 36.5 \\
\hline Economic situation & 118 & 60.8 \\
\hline IGA & & 39.2 \\
\hline No IGA & 197 & \\
\hline Number of pregnancies & 127 & \\
\hline$<2$ & & \\
\hline$\geq 2$ & & \\
\hline IGA: Income generating activity; $n:$ Number; \%: The frequency for each data. & \\
\hline
\end{tabular}

Proportions and odds ratio estimates were reported with their 95\% CIs. Sample sizes and type, HPV prevalence and genotypes, and single- and multiple-infection profiles were described. STATA software version 14.1 (StataCorp, TX, USA) was used for the statistical analyses, which were performed at a 5\% significance level. A logistic regression model was used for univariate and multivariate analyses.

\section{Ethical considerations}

Ethical approval for this study was obtained from the 'Comité de Bioéthique pour la Recherche en Santé (CBRS)' (Bioethics Committee for Health Research) of the Ministry of Health of Togo $\left(n^{\circ} 751 / 2014 / M S / C A B / D G S / D P L E T / C B R S\right)$. Participants provided written consent prior to participation and authorizations from the directors of selected medical centers were obtained before conducting this study.

\section{Results}

Socio-demographic \& clinical characteristics of the participants

A total of $341 \mathrm{HIV}$-negative women attending ordinary gynecological consultations were invited to participate in the study. A total of 15 (4.4\%) women refused to be sampled for the HPV test and were not included in the study. Two samples were damaged and were not analyzed. Finally, a total of 324 women were enrolled in this study. The median age of the study participants was 38 years old (interquartile range [IQR]: 29-47). Three-quarters (75\%) of the study participants had at least secondary education and $49.3 \%$ were married or cohabiting. More than half of the women $(60.8 \%)$ had fewer than two pregnancies (Table 1$)$.

\section{Prevalence of HPV infection}

$\mathrm{HPV}$ was found in 30 women, corresponding to a prevalence of HPV infection of 9.3\% (95\% CI: 6.3-12.39). HPV infection was more common in women $40-49$ years of age (14/102; 13.7\%), 20-29 years of age (9/89; 10.1\%), with a secondary $(16 / 154 ; 10.3 \%)$ or university $(9 / 89 ; 10.1 \%)$ education level, with no income generating activities $(15 / 118 ; 12.7 \%)$ and among those living alone $(18 / 164 ; 10.9 \%)$. However, these findings were not statistically significant ( $\mathrm{p}>0.05$; Table 2).

The prevalence of HR-HPV was 8.3\% (27/324) (95\% CI: 5.6-11.9). HR-HPV were more common among women aged $40-49$ years $(12 / 102 ; 11.7 \%), 20-29$ years $(8 / 89 ; 8.9 \%)$, those living alone $(16 / 164 ; 9.7 \%)$ and those with university degrees $(9 / 89 ; 10.1 \%)$, but the differences were not statistically significant $(\mathrm{p}>0.05$; Table 2$)$. 
Table 2. Socio-demographic, clinical and biological characteristics according to HPV type.

\begin{tabular}{|c|c|c|c|c|c|c|c|c|c|c|}
\hline \multirow[t]{2}{*}{ Characteristics } & \multirow{2}{*}{$\begin{array}{l}\text { Total } \\
(n=324) \\
n\end{array}$} & \multicolumn{3}{|c|}{${ }^{\ddagger} H P V+(n=30)$} & \multicolumn{3}{|c|}{${ }^{8}$ HR-HPV+ $(n=27)$} & \multicolumn{3}{|c|}{ I LR-HPV+ $(n=7)$} \\
\hline & & $n$ & $\%$ & p-value & $n$ & $\%$ & $p$-value & $n$ & $\%$ & $p$-value \\
\hline Age (years) & & & & 0.126 & & & 0.273 & & & 0.433 \\
\hline $20-29$ & 89 & 9 & 10.1 & & 8 & 8.9 & & 3 & 3.3 & \\
\hline $30-39$ & 80 & 3 & 3.7 & & 3 & 3.7 & & 0 & 0.0 & \\
\hline $40-49$ & 102 & 14 & 13.7 & & 12 & 11.7 & & 3 & 2.9 & \\
\hline$\geq 50$ & 53 & 4 & 7.5 & & 4 & 7.5 & & 1 & 1.8 & \\
\hline Marital status & & & & 0.374 & & & 0.461 & & & 0.513 \\
\hline Lives alone & 164 & 18 & 10.9 & & 16 & 9.7 & & 4 & 2.4 & \\
\hline Married/cohabiting & 160 & 12 & 7.5 & & 11 & 6.8 & & 3 & 1.8 & \\
\hline Education level & & & & 0.540 & & & 0.648 & & & 0.324 \\
\hline None or primary & 76 & 5 & 6.1 & & 5 & 6.1 & & 0 & 0.9 & \\
\hline Secondary & 138 & 16 & 10.3 & & 13 & 8.4 & & 4 & 3.5 & \\
\hline University & 80 & 9 & 10.1 & & 9 & 10.1 & & 3 & 3.7 & \\
\hline Economic situation & & & & 0.154 & & & 0.125 & & & 0.500 \\
\hline $\mathrm{IGA}^{\dagger}$ & 206 & 15 & 7.2 & & 13 & 6.3 & & 4 & 1.9 & \\
\hline No IGA & 118 & 15 & 12.7 & & 14 & 11.8 & & 3 & 2.5 & \\
\hline Number of pregnancies & & & & 0.200 & & & 0.204 & & & 0.436 \\
\hline$<2$ & 197 & 22 & 11.1 & & 20 & 10.1 & & 5 & 2.5 & \\
\hline$\geq 2$ & 127 & 8 & 6.2 & & 7 & 5.5 & & 2 & 1.5 & \\
\hline
\end{tabular}

Among HPV-infected women, HR-HPV was observed in $90.0 \%(27 / 30)$ and LR-HPV in $23.3 \%(7 / 30)$ (Table 2).

\section{HPV genotypes distribution}

Among the 30 women infected with HPV, HPV18 (15/30; 50\%) was the most common genotype identified, followed by HPV82 and HPV45, 62/81 (3/30; 10\%). Concomitant infection with both HPV16 and HPV18 was observed in one woman $(1 / 30 ; 3.3 \%)$. However, infection with HPV 11 or concomitant infection with HPV6 and HPV16 or HPV18 was not observed.

A total of 13 different HPV genotypes HR-HPV $(16,18,35,45,52,53,68,82)$ and LR-HPV $(6,40,43$, 44/65, 62/81) were detected (Figure 1).

Single and multiple infections were observed in $80 \%(24 / 30)$ and $20 \%(6 / 30)$ of participants, respectively. Among women infected with HR-HPV, 77.7\% (21/27) had a single infection $(16,18,35,52,53,68$ and 82), $3.7 \%(1 / 27)$ had an infection with two HR-HPV $(16,18)$ and $23.8 \%(5 / 21)$ had one HR-HPV and at least one LR-HPV $(40,43,44 / 65,62 / 81)$. Of oncogenic strains identified, the Cervarix ${ }^{\circledR}$ and Gardasil- $9^{\circledR}$ vaccines covered two strains for $53.3 \%$ and four strains for $70 \%$ of the participants, respectively.

\section{Factors associated with HR-HPV infection}

In univariate analyses, none of the explanatory variables were associated with HR-HPV infection (Table 3).

\section{Discussion}

In the present study, the prevalence of any-type HPV was relatively low $(9.3 \%)$ and this is comparable to the overall prevalence reported in Ghana (10.7\%), in Egypt (10.4\%, 10.3\%) and Northern Africa (10.9\%) [16,17]. It is one of the lowest prevalence reported in sub-Saharan Africa. Indeed, in a meta-analysis of 1 million women with normal cytological findings published in 2010, Bruni et al. reported a prevalence of $24 \%$ in sub-Saharan Africa [17]. This prevalence is also low compared with the prevalence found in other West African countries, with $33.2 \%$ in Benin, $24.3 \%$ in Burkina Faso and 50.3\% in Côte d'Ivoire $[5,9,18]$. This difference could be explained by many factors 


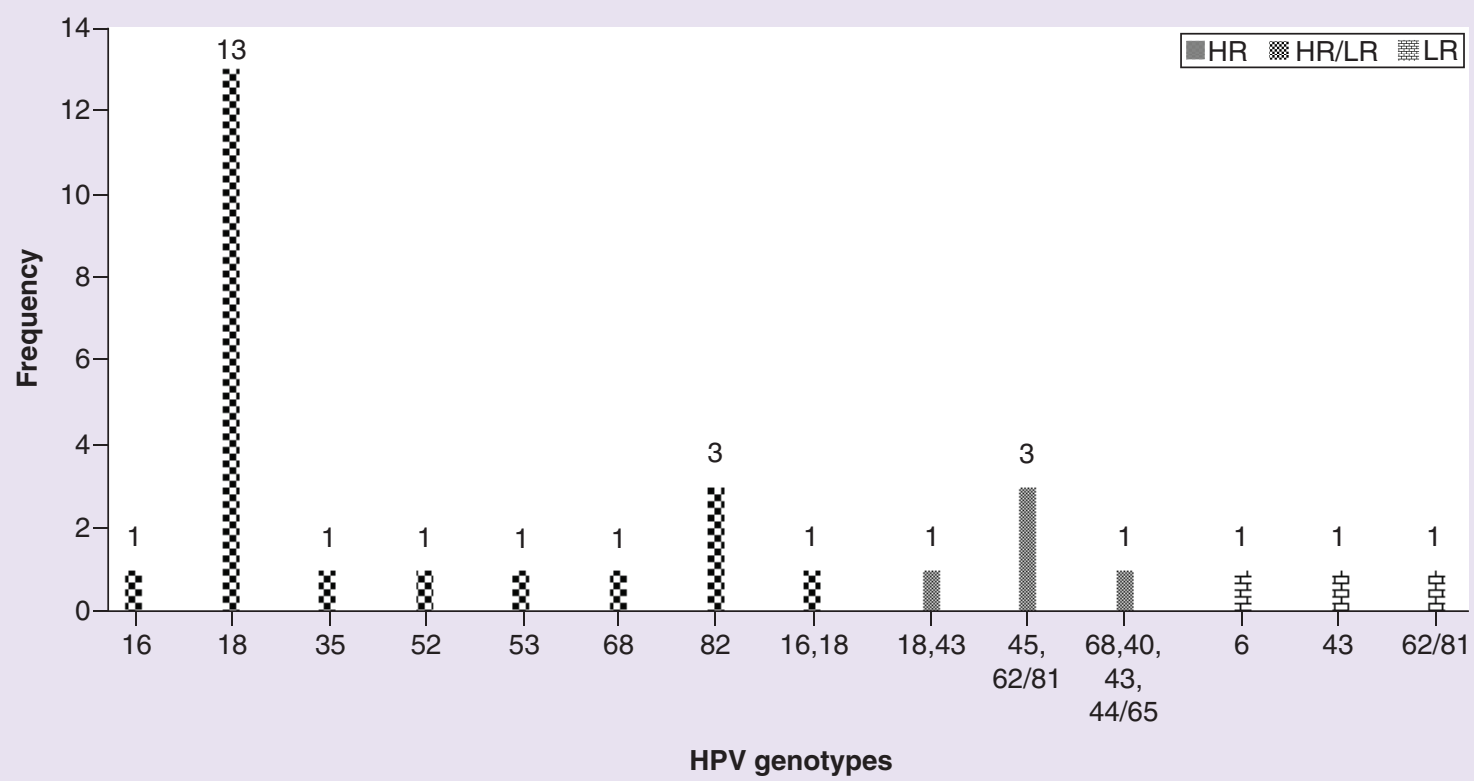

Figure 1. Human papillomavirus genotypes.

HPV: Human papillomavirus; HR: High risk; LR: Low risk.

\section{Table 3. Factors associated with HR-HPV infection.}

Characteristics

$\begin{array}{llllll} & \text { No } & \text { Yes } & \text { OR } & 95 \% \mathrm{Cl} & \mathrm{p} \text {-value }\end{array}$

Age (years)

$<35$

$\geq 35$

122

N

$\%$

0.683

\section{Marital status}

175

$\begin{array}{ll}41.1 & 10 \\ 58.9 & 17\end{array}$

Lives alone

Married/cohabiting

University level

Yes 80

No

Economic situation

IGA

No IGA

Number of pregnancies

$<2 \quad 17$

$\geq 2 \quad 120$

$120 \quad 40.4$

$\begin{array}{lll}148 & 49.8 & 16 \\ 149 & 50.2 & 11\end{array}$

37.0

63.0

1

$0.53-2.77$

$p>5 \%$ indicates no statistical difference.

\%: The prevalence in each specified category; HR-HPV: High-risk HPV; IGA: Income generating activities; n: Number; OR: Odds ratio.

such as the laboratory technique used, the age and HIV status of the population. In our population, all women were HIV-negative.

In our study, two population groups aged $20-29$ years $(10.1 \%)$ and $40-49$ years $(13.7 \%)$ had higher prevalence of HPV. Similar findings, with both peaks of age, have been documented in other studies $[11,17,19]$.

The prevalence of HR-HPV was $8.3 \%$. HR-HPV were more common among women aged $40-49$ years $(11.7 \%)$, living alone (9.7\%) and with a university degree (10.1\%). In Togo, high prevalence of HR-HPV has been previously 
reported in key populations, with $44 \%$ among men having sex with men, $32.9 \%$ among female sex workers and $16.7 \%$ in HIV-infected women $[8,11,12]$.

A total of 13 different HPV genotypes HR-HPV $(16,18,35,45,52,53,68,82)$ and LR-HPV (6, 40, 43, $44 / 65,62 / 81$ ) were found in the study's population. The identification of different genotypes, proves that there is a cocirculation of multiple HPV types in Lomé, Togo. Among the $30 \mathrm{HPV}$ infected women, the most common HPV types were HPV18 (50\%), HPV82 (10\%) and HPV45 (10\%). This distribution of HPV in Togo is consistent with literature data, in the general population for early diagnosis. HPV16 is the most common reported genotype in cancer or precancerous lesions [17-19]. Our results align with findings reported in Burkina Faso and in Ghana, neighboring countries in Togo, which found more HPV18 than HPV16 [9,20,21].

HPV35 found in our study was also found among men having sex with men and female sex workers in Togo, and in women of child-bearing age in Abidjan, Côte d'Ivoire [5,8,12]. Therefore, it is important to identify circulating genotypes for better management and protection of populations against HPV infections.

Some factors that could be responsible for these variations include the type of assay used, differences in population, multiple HPV infections and varying exposures of individuals to different risk factors [5]. Thus, the comparison of the prevalence between studies should be made with caution.

There are more single HPV infections than multiple HPV infections in this study. Infection with multiple HPV genotypes, which was found to be associated with an increased risk of HPV persistence [22], was relatively low in the present study. Furthermore, these findings are comparable to those of Ouedraogo and Traore et al. in Burkina Faso, Piras et al. in Benin and Krings et al. in Ghana [9,18,19,21]. Low detection of multiple HPV infections could be influenced by the specificity of the tests used for screening or diagnosis, especially if the test does not cover most genotypes circulating in the geographical area. In our study, we used a PCR and hybridization to detect the HPV DNA (18 HR and 18 LR-HPV) [14].

In this study, neither HR-HPV nor any type HPV infection was associated with age, marital status, education, economic situation and the number of pregnancies. This could be explained by the low prevalence of HPV reported in our study. However, the prevalence of genital HR-HPV is also directly related to some risk factors [2,22]. These risk factors could be responsible for HPV acquisition, persistence and development of cervical cancer. These risk factors can be early onset of sexual activity, low socioeconomic status, illiteracy, multiple sexual partners, marital status, alcoholism, smoking and use of oral contraceptives [5,9,19,22]. People who are immunocompromised such as those living with HIV or who have coinfection with other sexually transmitted agents are also more likely to have persistent HPV infections and a more rapid progression to precancer and cancer $[5,8,12]$. In a previous study among HIV infected women, a prevalence of $22.2 \%$ was reported in Togo [11].

This study presents some limitations. First, we recruited participants in clinical setting and findings from our study may not be generalized to all HIV-negative women of child bearing age in Togo. Second, cytological analyses of cervical swabs were not available to assess the association between HPV infection and clinical lesions. Third, as this study focuses more on the prevalence of HPV, sexual factors were not documented, such as the age at first intercourse, sexual partners and use of hormonal contraceptives. Hence, we did not have a broad set of factors for the analysis of risk factors.

Despite these limitations, to our knowledge, this was the first study reporting the prevalence of HPV infection and its genotype distribution among HIV-negative women of child-bearing age in Lomé, Togo. The previous studies were conducted among key populations (men who have sex with men and female sex workers). Further studies should be carried out among adolescents in order to have a complete distribution of HPV in Togo.

\section{Conclusion}

In our study, in HIV-negative women of child-bearing age in Togo, we found that one in ten women has an HPV infection. It is advisable to look for possible cross-reactions of the vaccine strains used in order to better protect the population against strains circulating in the country. Therefore, we strongly call for a program to screen for HPV in Togo. It is also urgent to introduce vaccines against HPV and propose a program against cervical cancer in Togo. 
and DK Ekouevi. Funding acquisition was performed by M Prince-David and A Dagnra. Investigation was performed by YT Nyasenu, FA Gbeasor-Komlanvi, DK Ekouevi and A Dagnra. Methodology was performed by YT Nyasenu, A Ehlan, FA Gbeasor-Komlanvi, MK Tchankoni, BM Yambiyo, M Salou and DK Ekouevi. Project administration was performed by YT Nyasenu, A Ehlan, M PrinceDavid and A Dagnra. Supervision was performed by A Dagnra, M Salou, A Ehlan and DK Ekouevi. Validation was performed by YT Nyasenu, FA Gbeasor-Komlanvi, A Ehlan, M Prince-David, M Salou, DK Ekouevi and A Dagnra. Visualization was performed by A Ehlan, FA Gbeasor-Komlanvi, DK Ekouevi and A Dagnra. YT Nyasenu, FA Gbeasor-Komlanvi and DK Ekouevi wrote the original draft. Writing, reviewing and editing was performed by YT Nyasenu, FA Gbeasor-Komlanvi, DK Ekouevi, A Ehlan, M Salou, M Prince-David and A Dagnra.

\section{Acknowledgments}

The authors express their gratitude to the Ministry of Health and Social Protection of Togo for funding this project. We extend our deepest gratitude to all the women who participated in this study and to the directors of selected medical centers in which the study was conducted.

Financial \& competing interests disclosure

This study was supported by the Ministry of Health and Social Protection of Togo. The funder did not have any influence on the contents or outcomes of the study nor in the decision to publish. The authors have no other relevant affiliations or financial involvement with any organization or entity with a financial interest in or financial conflict with the subject matter or materials discussed in the manuscript apart from those disclosed.

No writing assistance was utilized in the production of this manuscript.

\section{Ethical conduct of research}

The authors state that they have obtained appropriate institutional review board approval or have followed the principles outlined in the Declaration of Helsinki for all human or animal experimental investigations. In addition, for investigations involving human subjects, informed consent has been obtained from the participants involved.

\section{Open access}

This work is licensed under the Attribution-NonCommercial-NoDerivatives 4.0 Unported License. To view a copy of this license, visit http://creativecommons.org/licenses/by-nc-nd/4.0/

\section{Summary points}

- HPV has been recognized as being involved in $99.7 \%$ of cervical cancer. Cervical cancer is the second most frequent female cancers worldwide and in Togo, with an estimated mortality rate of $12.5 \%$ in Togo in 2018. Limited data on HPV are available among HIV-negative women in Togo.

- A cross-sectional study was conducted from April 2014 to September 2015, among HIV-negative women attending gynecological consultation in six health centers in Lomé. Cervical swabs were collected among women who gave their informed consent to participate in the study. HPV test was performed using HPV Direct Flow Chip.

- A total of 324 women were included.

- The prevalence of any type and oncogenic HPV was 9.3 and $8.3 \%$, respectively.

- Among the 30 women infected with HPV, HPV18 (15/30; 50\%) was the most common genotype identified, followed by HPV82 (3/30; 10\%) and HPV45 (3/30; 10\%).

- A total of 13 different genotypes HPV, high risk $(16,18,35,45,52,53,68,82)$ and low risk $(6,40,43,44 / 65$, 62/81), were detected.

- Single and multiples infections were observed in $80 \%(24 / 30)$ and $20 \%(6 / 30)$ of participants, respectively.

- In univariate analyses, there are no socio-demographic and clinical factors associated with high-risk HPV.

- Findings from this study provide essential insights for planning future public health strategies and for the HPV vaccination program.

\section{References}

1. Faridi R, Zahra A, Khan K, Idrees M. Oncogenic potential of human papillomavirus (HPV) and its relation with cervical cancer. Virol. J. 8(1), 269 (2011).

2. Ferlay J, Colombet M, Soerjomataram I et al. Estimating the global cancer incidence and mortality in 2018: GLOBOCAN sources and methods. Int. J. Cancer 144(8), 1941-1953 (2019). 
3. Lissouba P, Van de Perre P, Auvert B. Association of genital human papillomavirus infection with HIV acquisition: a systematic review and meta-analysis. Sex. Transm. Infect. 89(5), 350-356 (2013).

4. Bansal A, Singh MP, Rai B. Human papillomavirus-associated cancers: a growing global problem. Int. J. Appl. Basic Med. Res. 6(2), 84-89 (2016).

5. Jaquet A, Horo A, Charbonneau V et al. Cervical human papillomavirus and HIV infection in women of child-bearing age in Abidjan, Côte d'Ivoire, 2010. Br. J. Cancer 107(3), 556-563 (2012).

6. Joura EA, Giuliano AR, Iversen O-E et al. A 9-valent HPV vaccine against infection and intraepithelial neoplasia in women. N. Engl. J. Med. 372(8), 711-723 (2015).

7. Giuliano AR, Palefsky JM, Goldstone $S$ et al. Efficacy of quadrivalent HPV vaccine against HPV Infection and disease in males. N. Engl. J. Med. 364(5), 401-411 (2011).

8. Ferré VM, Gbeasor-Komlanvi FA, Collin G et al. Prevalence of human papillomavirus, HIV and other sexually transmitted infections among men having sex with men in Togo: a national cross-sectional survey. Clin. Infect. Dis. 69(6), 1019-1026 (2019).

9. Ouedraogo CMR, Djigma FW, Bisseye $\mathrm{C}$ et al. Epidemiology, characterization of genotypes of human papillomavirus in a population of women in Ouagadougou. J. Gynecol. Obstet. Biol. Reprod. 40(7), 633-638 (2011).

10. Amégbor K, Darre T, Padaro E, Tengué K, Abalo A, Napo-Koura G. Cancers in Togo from 1984 to 2008: epidemiological and pathological aspects of 5251 cases. J. Cancer Epidemiol. 2011, 319872 (2011).

11. Nyasenu YT, Gbeasor-Komlanvi FA, Ehlan A et al. Prevalence and distribution of Human Papillomavirus (HPV) genotypes among HIV infected women in Lomé, Togo. PLoS ONE 14(2), e0212516 (2019).

12. Ferré VM, Ekouevi DK, Gbeasor-Komlanvi FA et al. Prevalence of human papillomavirus, human immunodeficiency virus and other sexually transmitted infections among female sex workers in Togo: a national cross-sectional survey. Clin. Microbiol. Infect. 25(12), 1560 (2019).

13. Herraez-Hernandez E, Preda O, Alonso S, Pardo RS, Olmo A. Detection and genotyping of human papillomavirus DNA in formalin-fixed paraffin-embedded specimens with the HPV direct flow CHIP system. Open Virol. J. 7, 91-95 (2013).

14. Herraez-Hernandez E, Alvarez-Perez M, Navarro-Bustos G et al. HPV direct flow CHIP: a new human papillomavirus genotyping method based on direct PCR from crude-cell extracts. J. Virol. Methods 193(1), 9-17 (2013).

15. Eklund C, Forslund O, Wallin K-L, Dillner J. Global improvement in genotyping of human papillomavirus DNA: the $2011 \mathrm{HPV}$ LabNet International Proficiency study. J. Clin. Microbiol. 52(2), 449-459 (2014).

16. Shaltout MF, Sallam HN, AbouSeeda M et al. Prevalence and type distribution of human papillomavirus among women older than 18 years in Egypt: a multicenter, observational study. Int. J. Infect. Dis. 29, 226-231 (2014).

17. Bruni L, Diaz M, Castellsagué M, Ferrer E, Bosch FX, de Sanjosé S. Cervical human papillomavirus prevalence in 5 continents: meta-analysis of 1 million women with normal cytological findings. J. Infect. Dis. 202(12), 1789-1799 (2010).

18. Piras F, Piga M, De Montis A et al. Prevalence of human papillomavirus infection in women in Benin, West Africa. Virol. J. 8, 514 (2011).

19. Krings A, Dunyo P, Pesic A et al. Characterization of human papillomavirus prevalence and risk factors to guide cervical cancer screening in the north Tongu district, Ghana. PLoS ONE 14(6), e0218762 (2019).

20. Awua AK, Sackey ST, Osei YD, Asmah RH, Wiredu EK. Prevalence of human papillomavirus genotypes among women with cervical cancer in Ghana. Infect. Agent. Cancer 11(1), 4 (2016).

21. Traore IMA, Zohoncon TM, Dembele A et al. Molecular characterization of high-risk human papillomavirus in women in Bobo-Dioulasso, Burkina Faso. Biomed. Res. Int. 2016, 7092583 (2016).

22. Ingabire C, Lim MK, Won Y-J, Oh J-K. Human papillomavirus genotype-specific persistence and potential risk factors among Korean women: results from a 2-year follow-up study. Cancer Res. Treat. 50(3), 813-822 (2017). 